\title{
Manejo de la Cetoacidosis Diabética en un hospital universitario de Montevideo en el período Junio - Setiembre de 2015.
}

Santiago Cabral ORCID: 0000-0001-7337-0473 Bachiller Medicina. Ayudante de Clase. Departamento de Farmacología y Terapéutica.

Noelia Speranza ORCID: 0000-0002-3330-9974 Médico Pediatra. Farmacóloga. Profesor Agregado Departamento de Farmacología y Terapéutica.

Stephanie Viroga ORCID: 0000-0001-9065-6692 Médico Ginecóloga. Farmacóloga. Profesor Adjunta Departamento de Farmacología y Terapéutica.

Gustavo Tamosiunas ORCID: 0000-0002-3619-9651

Médico Cardiólogo. Farmacólogo. Profesor Director Departamento de Farmacología y Terapéutica.

Ernesto Balverde ORCID: 0000-0001-7228-8646 Médico.

Soledad Herrera ORCID: 0000-0003-0708-3366 Médico.

Leticia González ORCID: 0000-0001-6694-9815 Médico.

María Eugenia Hernández ORCID: 0000-0003-0487-0102 Médico.

María Joaquina Goldaraz ORCID: 0000-0001-7023-9971 Médico.
Management of Diabetic Ketoacidosis in a Montevideo university hospital in the period June-September 2015

\section{Tratamento da cetoacidose diabética em um hospital universitário de} Montevidéu no período de Junho a Setembro de 2015

Resumen: Introducción. La cetoacidosis diabética es una complicación aguda frecuente y seria de la diabetes mellitus, suponiendo $5 \%$ de su mortalidad. Existe evidencia internacional que la adherencia a las guías de tratamiento de la cetoacidosis diabética, disminuye su morbimortalidad y que no existe un cumplimiento riguroso de dichas guías. El objetivo del trabajo fue evaluar la adecuación a las pautas de tratamiento de la cetoacidosis diabética de la American Diabetes Association, en la puerta de emergencia. Metodología. Se realizó un estudio observacional descriptivo del tratamiento realizado en la emergencia de un Hospital universitario de Montevideo a los pacientes con diagnóstico de cetoacidosis diabética, entre junio y setiembre de 2015. Se comparó la terapéutica aplicada con el protocolo de manejo propuesto por la American Diabetes Association en 2009. Resultados. Un total de 17 casos fueron incluidos en el análisis. El diagnóstico fue correcto en 9 pacientes, el tratamiento en la primera hora fue completo en 7 pacientes y la monitorización paraclínica en las primeras 6 horas fue completa en 3 pacientes. Conclusión. Se evidenció adecuación aceptable a las guías en la mayoría de los casos.

Palabras clave: Cetoacidosis Diabética, Guías de Práctica Clínica como Asunto, Insulina.

Abstract: Introduction. Diabetic ketoacidosis is a frequent and serious acute complication of diabetes mellitus, accounting for $5 \%$ of its mortality. There is international evidence that adherence to the treatment guidelines for diabetic ketoacidosis decreases its morbidity and mortality and that there is no rigorous compliance with these guidelines. The objective of the study was to evaluate the adequacy of the diabetic ketoacidosis treatment guidelines of the American Diabetes Association, at the emergency door.Methodology. A descriptive observational study was carried out on the treatment carried out in the emergency room of a Montevideo university hospital for patients diagnosed with diabetic ketoacidosis, between June and September 2015. The applied therapy was compared with the management protocol proposed by the American Diabetes Association in 2009. Results. A total of 17 cases were included in the analysis. The diagnosis was correct in 9 patients, the treatment in the first hour was complete in 7 patients and the paraclinical monitoring in the first 6 hours was complete in 3 patients. Conclusion. Acceptable adequacy to the guidelines was evidenced in most cases.

Key words: Diabetic Ketoacidosis, Practice Guidelines as Topic, Insulin.

Resumo: Introdução. A cetoacidose diabética é uma complicação aguda frequente e grave do diabetes mellitus, sendo responsável por $5 \%$ de sua mortalidade. Há evidências internacionais de que a adesão às diretrizes de tratamento para cetoacidose diabética diminui sua morbidade e mortalidade e que não há conformidade rigorosa com essas diretrizes. O objetivo do estudo foi 
avaliar a adequação das diretrizes de tratamento da cetoacidose diabética da American Diabetes Association, na porta de emergência. Metodologia. Foi realizado um estudo observacional descritivo sobre o tratamento realizado no pronto-socorro de um hospital universitário de Montevidéu para pacientes com diagnóstico de cetoacidose diabética, entre junho e setembro de 2015. A terapia aplicada foi comparada com o protocolo de manejo proposto pela American Diabetes Association em 2009. Resultados. Um total de 17 casos foram incluídos na análise. O diagnóstico foi correto em 9 pacientes, o tratamento na primeira hora foi completo em 7 pacientes e o monitoramento paraclínico nas primeiras 6 horas foi completo em 3 pacientes. Conclusão. A adequação aceitável às diretrizes foi evidenciada na maioria dos casos.

Palavras-chave: Cetoacidose Diabética, Guias de Prática Clínica como Assunto, Insulina. 


\section{Introducción}

La diabetes mellitus (DM) es una enfermedad crónica de origen multifactorial que constituye un problema de salud pública debido a su alta prevalencia y gravedad de sus complicaciones.

El Center for Disease Control and Prevention (CDC) en su National Diabetes Statistics Report publicado en 2020 estimó una prevalencia de DM cercana a 13\% de la población total correspondiente a 34 millones de estadounidenses mayores de 18 años, alcanzando $27 \%$ de prevalencia si se considera únicamente a los mayores de 65 años ${ }^{(1)}$. En Uruguay la segunda encuesta nacional de factores de riesgo de enfermedades no transmisibles realizada en 2013 reportó que la prevalencia de DM es de $6 \%$ y en concordancia con el reporte del CDC, la prevalencia aumenta con la edad, alcanzando $17 \%$ si se considera únicamente a los mayores de 55 años (2).

La cetoacidosis diabética (CAD) es una complicación aguda frecuente de la diabetes. Los datos reportados por el CDC estiman una tasa de consultas en emergencia de 8.8 por 1000/ habitantes en el año $2016^{\left({ }^{1)}\right.}$. La tasa de mortalidad global por CAD es de $4 \%{ }^{(3)}$. Esta aumenta con la edad reportándose una tasa de mortalidad de $22 \%$ en adultos mayores de 65 años versus $2 \%$ en pacientes de entre 18 y 65 años ${ }^{(4)}$. La CAD es la principal causa de muerte en niños y adolescentes con Diabetes tipo 1 , siendo responsable del $50 \%$ de los fallecimientos en esta población ${ }^{(5)}$.

La CAD debe sospecharse en todo paciente con hiperglicemia. La tríada diagnóstica consiste en hiperglicemia (glicemia $>250 \mathrm{mg} / \mathrm{dL}$ ), hipercetonemia (acetoacetato y/o $\beta$-hidroxibutirato positivo) y acidosis metabólica $(\mathrm{pH}<7.3$ y HCO3- $<18 \mathrm{mEq} / \mathrm{L}){ }^{(5)}$. Siendo el criterio clave la hipercetonemia debido a que cerca del $10 \%$ de las CAD se presentan en euglicemia ${ }^{(5)}$. Además del $\mathrm{pH}$, el bicarbonato sérico, anión gap y la alteración del sensorio, permiten categorizar la CAD en leve, moderada o severa ${ }^{(5)}$.

Para reducir la variabilidad en el manejo de la CAD y mejorar los resultados clínicos se han desarrollado guías de práctica clínica basadas en evidencia.

Hara y cols demostraron que la aplicación de un protocolo de tratamiento de la CAD redujo significativamente el tiempo de resolución de la CAD de 20 a 12 horas y el tiempo de estadía hospitalaria de 5.4 a 3.8 días, sin diferencias significativas en la proporción de pacientes con hipoglicemia o hipopotasemia ${ }^{(6)}$.

Periódicamente la American Diabetes Association (ADA) publica su guía de manejo de la CAD ${ }^{(7)}$, siendo ampliamente aceptada por la comunidad científica como marco de referencia para el tratamiento de esta complicación del paciente diabético. Laliberte y cols compararon el antes y después de la implementación de la guía ADA 2009 en una unidad de cuidados intensivos y reportaron mejoría estadísticamente significativa en la reposición hídrica (de $27 \%$ a $71 \%$ ) e insulinoterapia (de $60 \%$ a $81 \%)^{(8)}$. Sin embargo el cumplimiento de las guías de tratamiento es bajo, con valores reportados de $20 \%$ por Fusco y cols en Estados Unidos ${ }^{(9)}$ o $30 \%$ reportado por Singh y cols en Reino Unido ${ }^{(10)}$.

En Uruguay no se conocen datos sobre cumplimiento de guías de práctica clínica de CAD. El objetivo de este estudio es evaluar el nivel de cumplimiento de las pautas de tratamiento de la CAD recomendadas por la ADA, en la puerta de emergencia de un hospital universitario, en el período de junio-setiembre de 2015.

\section{Metodología}

Se realizó un estudio observacional descriptivo del tratamiento realizado en la emergencia de un Hospital universitario de Montevideo, a los pacientes con diagnóstico médico de CAD en el período comprendido entre el $1^{\circ}$ de junio y el 30 de setiembre de 2015.

Se comparó la terapéutica aplicada con la recomendada por la guía ADA 2015.

Los criterios de inclusión para el estudio fueron: pacientes mayores de 15 años con diagnóstico de CAD por criterio del médico tratante, admitidos en la emergencia del hospital universitario, que aceptaran la participación en el trabajo mediante consentimiento informado. Fueron excluidos del estudio las pacientes embarazadas y aquellos que se negaron a participar.

No fue posible calcular el tamaño muestral dado que se desconoce la incidencia de CAD en nuestro medio y en el hospital. 
El método de notificación de los casos se basó en un sistema de alarma telefónica, mediante el cual el equipo de trabajo de la emergencia del hospital notificó a los investigadores sobre el ingreso de un nuevo paciente con diagnóstico de CAD a la puerta de emergencia. Frente a la alerta telefónica, uno de los investigadores concurrió a la emergencia a solicitar el consentimiento informado y los datos del paciente. Para esto se creó entre los investigadores un cronograma de guardias semanal. Para la organización y diseño de este sistema se realizaron reuniones de coordinación entre el equipo de emergencia del hospital y el equipo de investigación.

La recolección de los datos se realizó mediante la revisión de la historia clínica de emergencia del paciente ingresado, con posterior registro en una ficha de recolección de datos prediseñada.

\section{a) Variables en estudio}

La variable primaria fue el nivel de cumplimiento de las pautas de tratamiento de la CAD establecidas en la declaración de la ADA "Hyperglycemic crises in adult patients with diabetes" (7) publicada en 2009 y citado por la guía American Diabetes Association Standards of Medical Care In Diabetes $2015^{(11)}$. En el cuadro 1 se detallan los principales componentes de la declaración de la ADA.

Otra variable evaluada fue efectividad de las dos marcas comerciales de insulina regular disponibles en el hospital.

\begin{tabular}{|c|c|}
\hline \multicolumn{2}{|l|}{ Griterios Diagnósticos } \\
\hline Glicemia & $>250 \mathrm{mg} / \mathrm{dL}$ \\
\hline $\mathrm{pH}$ & Arterial $\leq 7.3$ \\
\hline Bicarbonato sérico & $\leq 18 \mathrm{mEq} / \mathrm{L}$ \\
\hline Cetonemia o cetonuria & Positiva \\
\hline \multicolumn{2}{|l|}{ Tratamiento } \\
\hline Terapia con fluidos & $\begin{array}{l}\text { Suero isotónico salino }(0.9 \% \mathrm{NaCl}) 1-1.5 \mathrm{~L} \text { en la primera hora. Luego } 250-500 \\
\mathrm{~mL} / \mathrm{h} \text { con suero } 0.45 \% \mathrm{NaCl} \text { cuando natremia normal o elevada o suero } 0.9 \% \\
\mathrm{NaCl} \text { cuando natremia disminuida. }\end{array}$ \\
\hline Insulinoterapia & $\begin{array}{l}\text { Insulina regular bolo i/v } 0.1 \mathrm{U} / \mathrm{kg} \text { seguido por } 0.1 \mathrm{U} / \mathrm{kg} / \mathrm{h} \text { infusión intravenosa } \\
\text { continua. Cuando glicemia }<200 \mathrm{mg} / \mathrm{dL} \text { disminuir la dosis a } 0.02-0.05 \mathrm{U} / \mathrm{kg} / \mathrm{h} \\
\text { y agregar dextrosa } 5 \% \text { a la terapia con fluidos. Objetivo de glicemia } 150-200 \\
\mathrm{mg} / \mathrm{dL} \text {. }\end{array}$ \\
\hline Potasio & $\begin{array}{l}\text { Si calemia }>5.0 \mathrm{mEq} / \mathrm{L} \text { no administrar potasio. Si calemia }<5.0 \mathrm{mEq} / \mathrm{L} \\
\text { administrar } 20-30 \mathrm{mEq} \text { por litro de suero. Si calemia }<3.3 \mathrm{mEq} / \mathrm{L} \text { administrar } \\
20-30 \mathrm{mEq} / \mathrm{L} \text { por litro de suero y retrasar el inicio de la insulinoterapia hasta } \\
\text { calemia }>3.3 \mathrm{mEq} / \mathrm{L} \text {. Objetivo de calemia entre } 4-5 \mathrm{mEq} / \mathrm{L} \text {. }\end{array}$ \\
\hline Bicarbonato & $\begin{array}{l}\text { Si pH > } 6.9 \text { no administrar HCO3-. Si pH }<6.9 \text { administrar NaHCO3 } 100 \mathrm{mEq}+ \\
20 \mathrm{mEq} \text { KCl diluido en } 400 \mathrm{~mL} \text { a una velocidad de } 200 \mathrm{~mL} / \mathrm{h} \text { por } 2 \text { hs o hasta } \\
\text { que } \mathrm{pH}>6.9 \text {. }\end{array}$ \\
\hline \multicolumn{2}{|l|}{ Monitorización } \\
\hline & $\begin{array}{l}\text { Gasometría, ionograma y glicemia en puerta de emergencia y repetir para } \\
\text { controlar respuesta y adecuar el tratamiento. }\end{array}$ \\
\hline \multicolumn{2}{|l|}{ Criterio de Resolución } \\
\hline & $\begin{array}{l}\text { Glicemia }<200 \mathrm{mg} / \mathrm{dL} \text { y dos de los siguientes criterios: bicarbonato sérico } \geq 15 \\
\mathrm{mEq} / \mathrm{L} \text {, } \mathrm{pH} \text { venoso }>7.3 \text { y anión gap calculado } \leq 12 \mathrm{mEq} / \mathrm{L} \text {. }\end{array}$ \\
\hline
\end{tabular}

\section{b) Operacionalización de las variables}

El nivel de adecuación a los criterios diagnósticos sugeridos por la ADA fue reportado como porcentaje de pacientes que cumplen con los cuatro criterios propuestos: glicemia $>250 \mathrm{mg} /$ $\mathrm{dL}, \mathrm{pH}$ arterial o venoso $\leq 7.3$, bicarbonato sérico $\leq 18 \mathrm{mEq} / \mathrm{L}$ y cetonemia o cetonuria positiva.

El cumplimiento de las pautas de tratamiento se clasificó en cumplimiento completo o cumplimiento aceptable. Se consideró cumplimiento completo al tratamiento con suero fisiológico 1-1.5 L en la primera hora de asistencia, insulina regular a dosis de $0.1 \mathrm{UI} / \mathrm{kg} / \mathrm{h}$ por infusión intravenosa continua, administración de potasio cuando $[\mathrm{K}+]<5.0 \mathrm{mmol} / \mathrm{L}$ y administración de bicarbonato cuando $\mathrm{pH}<6.9$. Se consideró cumplimiento aceptable al tratamiento con suero fisiológico 1-1.5 L en la primera hora e insulina regular a dosis de $0.1 \mathrm{Ul} / \mathrm{kg} / \mathrm{h}$. 
Para la monitorización paraclínica, la declaración de la ADA recomienda monitorización individualizada para cada paciente sin establecer un número específico de controles. Sin embargo, por practicidad se decidió establecer como monitorización paraclínica completa de un paciente la realización de un mínimo de 2 glicemias, 2 ionogramas y 2 gasometrías arteriales en las primeras 6 horas desde el ingreso a la puerta de emergencia. Este número de controles se determinó como el mínimo de controles necesarios para establecer un correcto tratamiento inicial y por lo menos un ajuste del tratamiento en las primeras horas del ingreso a la puerta de emergencia.

Se definió compensación de la CAD cuando un paciente tuvo una glicemia $<200 \mathrm{mg} / \mathrm{dL}$ y dos de los siguientes criterios: bicarbonato sérico $>15 \mathrm{mEq} / \mathrm{L}, \mathrm{pH}$ venoso $>7.3$ y anión gap calculado $\leq 12 \mathrm{mEq} / \mathrm{L}$, acorde a lo establecido en la ADA 2015 (11).

Durante el período de estudio la marca de insulina regular disponible en la puerta de emergencia fue rotando en períodos de una semana. Los autores del trabajo solicitaron que semanalmente desde la farmacia del hospital se entregara secuencialmente una y otra marca identificada como 1 o 2 para quienes recolectaban los datos. La efectividad fue expresada como proporción de pacientes que lograron la compensación antes de las 12 horas desde la administración de la primera dosis, tiempo en horas hasta la compensación y promedio de días de estadía en emergencia.

Las dos marcas comerciales de insulina regular disponibles en el hospital en el periodo de estudio fueron INSUBEST R® y WOSULIN R®. Para el análisis de efectividad entre las marcas de insulina se realizó una prueba de independencia de chi-cuadrado para evaluar la relación entre la marca de insulina y la compensación antes de las 12 horas desde la administración de la primera dosis de insulina. Además se realizó un contraste de hipótesis con la prueba estadística t de Student para evaluar la relación entre marca de insulina y tiempo hasta compensación.

\section{c) Consideraciones éticas}

El presente estudio fue aprobado por el comité de ética del Hospital universitario en 2015. Todos los pacientes que aceptaron participar firmaron un consentimiento informado. Todo el diseño e implementación de este estudio se realizó respetando las normas establecidas en la declaración de Helsinki del año 2000.

\section{Resultados}

Un total de 20 casos de CAD fueron atendidos en la puerta de emergencia del hospital durante el período de estudio. Tres casos fueron excluidos por registro incompleto de la ficha de recolección de datos, por lo que el total de casos incluidos en el estudio fue de 17 pacientes.

En la tabla 1 se describen sus características.

\begin{tabular}{|c|c|}
\hline Variable & \\
\hline Edad & \\
\hline \multirow{4}{*}{$\begin{array}{l}\text { Media y rango (años) } \\
\leq 18 \text { años (n) } \\
>18 \text { a } 35 \text { años (n) } \\
>35 \text { años (n) }\end{array}$} & $35.7(15-72)$ \\
\hline & 3 \\
\hline & 7 \\
\hline & 7 \\
\hline \multirow{3}{*}{$\begin{array}{l}\text { Tipo de DM } \\
\text { Tipo } 1 \text { (n) } \\
\text { Tipo } 2 \text { (n) }\end{array}$} & \\
\hline & 11 \\
\hline & 6 \\
\hline \multirow{3}{*}{$\begin{array}{l}\text { Presentación } \\
\text { Debut (n) } \\
\text { Descompensación (n) }\end{array}$} & \\
\hline & 1 \\
\hline & 16 \\
\hline
\end{tabular}

Los criterios diagnósticos de CAD utilizados por los médicos tratantes cumplieron los cuatro criterios establecidos por la ADA en un total de $9 / 17$ pacientes (53\% de los casos), en 5/17 pacientes alguno de los criterios no fue incluido en el diagnóstico y en 3/17 pacientes se hizo diagnóstico con solo un criterio bioquímico y la impresión clínica. 
En el gráfico 1 se presenta el cumplimiento del tratamiento.

Gráfico 1: Cumplimiento del tratamiento en los pacientes con CAD según criterios ADA.

Gráfico 2: Cumplimiento de los estudios paraclínicos en los pacientes con CAD según criterios ADA

Tabla 2: Diferencias entre marcas de insulina regular. $\mathrm{N}=15$

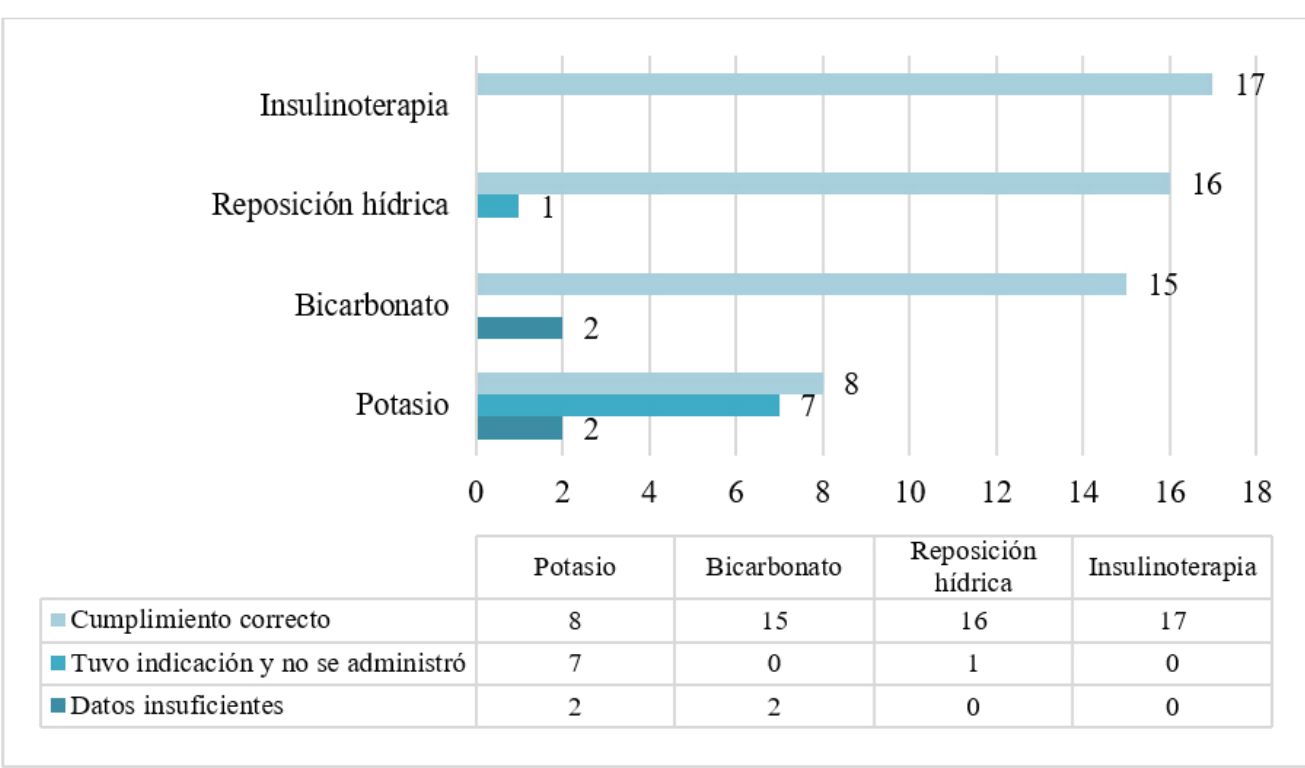

La monitorización paraclínica completa se realizó en 3/17 pacientes. En el Gráfico 2 se presentan los mismos. En 14 pacientes se realizaron al menos 2 glicemias en las primeras 6 horas, en 7 pacientes se realizaron al menos 2 gasometrías y en 5 pacientes se realizaron al menos 2 ionogramas en las primeras 6 horas.

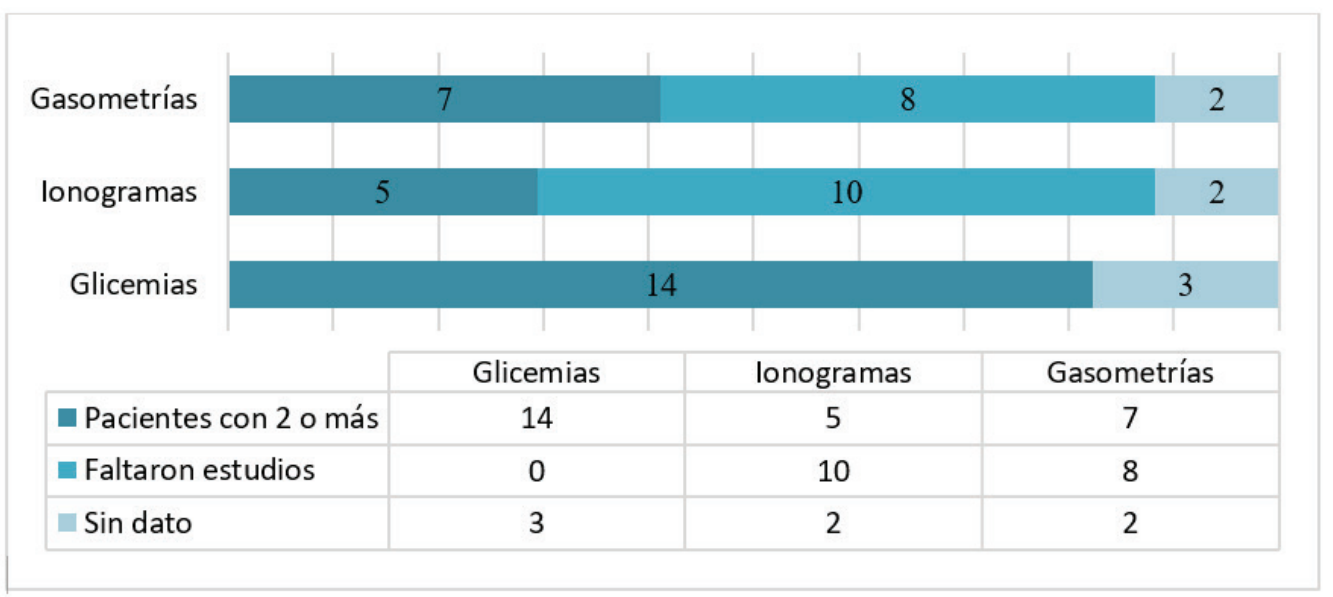

En la tabla 2 se presentan los resultados de análisis de efectividad entre las dos marcas de insulina. No se contó con registro de datos en 2 pacientes.

\begin{tabular}{|c|c|c|c|}
\hline Variable & INSUBEST R@ & WOSULIN R@ & \\
\hline \multirow{3}{*}{$\begin{array}{l}\text { Compensación < 12hs } \\
\text { Si (n) } \\
\text { No (n) }\end{array}$} & $\mathrm{n}=10$ & $\mathrm{n}=5$ & \\
\hline & 3 & 2 & \multirow{2}{*}{$X 2=0.15$} \\
\hline & 7 & 3 & \\
\hline \multirow{2}{*}{$\begin{array}{l}\text { Tiempo hasta compensación } \\
\text { Media (horas) }\end{array}$} & $n=8$ & $n=4$ & \multirow{2}{*}{$t=-0.063$} \\
\hline & 15 & 16 & \\
\hline \multirow{2}{*}{$\begin{array}{l}\text { Duración de estadía en emergencia } \\
\text { Media [rango] (días) }\end{array}$} & $n=10$ & $n=5$ & \\
\hline & $2.6[1-4]$ & $2.6[1-5]$ & \\
\hline
\end{tabular}

Un total de 10 pacientes fueron tratados con insulina regular INSUBEST R® y 5 con WOSULIN R®. La duración del ingreso en emergencia no tuvo diferencias entre marcas, con una media de 2.6 (rango 1-5) días de estadía para ambas. La proporción de pacientes compensados 
en las primeras 12 horas con cada marca no varió de forma significativa. El tiempo hasta la compensación tampoco varió de forma significativa entre marcas.

\section{Discusión}

La CAD posee un tratamiento dinámico que requiere monitorización y decisiones clínicas acertadas y tempranas. Estudios previos han mostrado que el uso de protocolos para el manejo de la CAD se asocia a mejores resultados, particularmente reducción en el tiempo de estadía hospitalaria ${ }^{(6)}$, pero no se han reportado datos sobre el cumplimiento de dichos protocolos a nivel local.

La proporción de pacientes con diagnóstico de CAD que cumplieron con los cuatro criterios establecidos por la ADA fue aceptable. Se destaca que un paciente no cumplió con dichos criterios debido a glicemia $<250 \mathrm{mg} / \mathrm{dL}$, situación que ocurre en $10 \%$ de los pacientes con CAD. Este resultado se corresponde con el reportado por Devalia y cols donde al evaluar el cumplimiento del protocolo británico Joint British Diabetes Societies for Inpatient Care en un hospital de Inglaterra, se diagnosticó correctamente a $78 \%$ de los pacientes con CAD ${ }^{(12)}$.

La CAD en euglicemia se ha reportado en pacientes embarazadas (no incluidas en este estudio), pacientes con afectación de la gluconeogénesis por daño hepático y en pacientes tratados con inhibidores del cotransportador sodio-glucosa de tipo 2, también se puede explicar por inicio extra-hospitalario de la terapéutica previo al ingreso a emergencia ${ }^{(5)}$.

El bajo porcentaje de casos en los que hubo una correcta monitorización en el presente estudio, también coincide con los resultados del estudio de Devalia, quien reporta una monitorización plasmática completa entre la 2 da y 4 ta hora de tratamiento del $38 \%{ }^{(12)}$. Este resultado podría explicarse por sobrecarga laboral de los médicos asistenciales en las puertas de emergencia con gran número de pacientes a cargo de cada uno, además los protocolos de tratamiento de la CAD pueden no haberse utilizado como check-list para asegurar un correcto cumplimiento o podría deberse a que el juicio clínico de cada médico frente a cada situación fue determinante a la hora de decidir los exámenes paraclínicos a realizar.

El análisis de la efectividad entre las marcas comerciales de insulina regular sintética INSUBEST R® y WOSULIN R® no mostró diferencias significativas cuando esta se evaluó como proporción de pacientes que lograron la resolución de la CAD en las primeras 12 horas o tiempo hasta que se logró la compensación. No se encontraron estudios de efectividad entre marcas comerciales como para comparar con los resultados hallados en este estudio.

Este hallazgo es interesante dado que es un hecho frecuente en la práctica clínica aducir problemas de efectividad a la marca comercial de un medicamento. En el caso de las insulinas que no requieren estudios de bioequivalencia, es importante poder comprender cuáles podrían ser los determinantes de falta de efectividad vinculados al medicamento, si no pueden atribuirse al principio activo. Por ejemplo, los dispositivos con los que se utilizan las insulinas (lapiceras y bombas de infusión) pueden presentar diferencias de calidad o puede haber desempeño variable con el uso de algoritmos de computadora para la regulación de la administración de insulina frente a la forma convencional manual ${ }^{(13,14)}$.

A pesar de no haber logrado buenos resultados en cumplimiento completo, la mayoría de los pacientes tuvieron un manejo aceptable, destacándose la correcta insulinoterapia en todos los casos. Estos resultados coinciden con los obtenidos por Dhatariya y cols quienes reportaron en 283 pacientes un cumplimiento de la reposición hídrica e insulinoterapia de 96.5\% y 91.5\% respectivamente, en la primera hora de tratamiento, según lo establecido en la guía británica de manejo de CAD ${ }^{(15)}$. Los pilares fundamentales de la terapéutica de los pacientes con CAD son la reposición hídrica y la insulinoterapia debido a que en conjunto restauran el volumen intravascular, la perfusión renal, los niveles de hormonas contrarreguladoras, la hiperglucemia, suprimen la lipólisis y la cetogénesis. Se deben administrar a todos los pacientes con CAD, mientras que la reposición de potasio y bicarbonato solo se administra cuando corresponde, de modo que el cumplimiento aceptable en la amplia mayoría de pacientes es un resultado favorable.

La reposición de potasio fue inadecuada en más de la mitad de los pacientes incluidos en el estudio, siendo el principal determinante del bajo cumplimiento completo del tratamiento de la CAD. En pacientes con CAD los niveles de potasio corporal total tienen un déficit de 3-5 mEq/ $\mathrm{kg}$, sin embargo los niveles de potasio séricos usualmente son normales o incluso elevados, debido a la salida del potasio intracelular inducida por la hipertonicidad, deficiencia de insulina y acidosis (5). Cieluch y cols evaluaron la adherencia al protocolo de reposición de potasio y su relación con la duración de la CAD en 242 pacientes y demostraron que la no adherencia al 
protocolo ocurrió en $71 \%$ de los pacientes y se asoció significativamente a mayor duración de la CAD y a mayor volumen de potasio transfundido ${ }^{(16)}$.

Este estudio muestra que se logró una adecuación completa al tratamiento recomendado por la ADA en menos de la mitad de los pacientes. Estos resultados son similares a los obtenidos por Singh y cols en un hospital de Escocia donde la adecuación del tratamiento a su protocolo institucional fue de $30 \%{ }^{\left({ }^{10}\right)}$. Este autor plantea dudas sobre la efectividad de la implementación de guías de práctica clínica como método para la estandarización del manejo de varias patologías. La atención se debería centrar en actividades que permitan mejorar la adecuación a las guías para poder evaluar su efectividad y detectar barreras que dificulten su cumplimiento.

Existen algunas limitaciones del presente estudio, como el bajo número de pacientes incluidos. Esto puede explicarse porque fue una muestra por conveniencia, delimitada al periodo de estudio, además, la captación de pacientes dependió de la notificación por parte del personal de la emergencia, el cual no está exento de olvidos. No es posible determinar si la incidencia de CAD es baja en nuestro país o si hubo fallas en el sistema de alarma. Esta muestra no permite realizar inferencia poblacional. Sería bueno contar con datos poblacionales al respecto. No haber calculado una muestra previamente para comparar la efectividad entre las dos marcas de insulina regular dificulta realizar conclusiones sobre los resultados hallados. No era el principal objetivo del estudio pero sería importante poder ampliar este tipo de investigaciones para analizar los determinantes de la falta de efectividad que pueden relacionarse con las marcas comerciales (como uno más de los muchos determinantes). Por otro lado, al utilizarse la historia clínica en papel como fuente de información, pudo omitirse terapéutica o monitorización que no haya sido registrada en la misma. Además en algunos casos fue difícil la interpretación de las indicaciones manuscritas en la historia clínica.

Como demostró Volkova y cols, el cumplimiento del protocolo de tratamiento de CAD aumentó cuando se introdujo el protocolo pero además se realizaron intervenciones educativas como discusión de casos, pruebas de conocimiento y lectura de artículos ${ }^{(17)}$. Los aspectos educativos continuos son fundamentales para optimizar las prácticas terapéuticas diarias.

Otra herramienta que podría mejorar la adecuación a las guías es tener estipulado la auditoría de historias clínicas de este cuadro clínico ${ }^{(18)}$. Uruguay ha implementado el desarrollo de la historia clínica electrónica nacional como estrategia de control, información y recordatorio, lo que constituye una herramienta potencial para mejorar la calidad del registro de datos, la estandarización de los procedimientos y la calidad asistencial (19).

\section{Conclusiones}

El nivel de cumplimiento de las recomendaciones de tratamiento de la CAD establecidas por la ADA fue aceptable en la mayoría de los casos y completo en menos de la mitad. Estos resultados no fueron afectados por la marca de insulina utilizada, si bien el número de pacientes incluidos fue pequeño.

Esta información justifica la realización de intervenciones educativas para el personal de salud con el objetivo de aumentar el grado de cumplimiento de las guías de tratamiento de la CAD. Este estudio puede utilizarse como comparación para futuros estudios que evalúen la efectividad de dichas intervenciones.

\section{Conflicto de interés}

Los autores declaran no tener conflictos de interés.

\section{Bibliografía}

1- Centers for Disease Control and Prevention. National Diabetes Statistics Report [sede Web]. Atlanta, GA: CDC; 2020 [actualizada el 2 de febrero de 2020; acceso 5 de mayo de 2020]. Disponible en: https://www.cdc.gov/diabetes/data/statistics-report/index.html

2- Uruguay. Ministerio de Salud Pública. Dirección Nacional de Salud. Segunda encuesta nacional de factores de riesgo de enfermedades no transmisibles [sede Web]. Montevideo: MSP; 2018 [actualizada el 03 de julio de 2018; acceso 5 de mayo de 2020]. Disponible en: https://www.gub.uy/ministeriosalud-publica/comunicacion/publicaciones/2da-encuesta-nacional-de-factores-de-riesgo-deenfermedades-no

3- Bhardwaj VR, Metcalfe N, Innes E, Harrison E, Jenkins D. Recurrent diabetic ketoacidosis after changing pen devices for insulin injection. BMJ. 2006 May 27;332(7552):1259-60. doi: 10.1136/ bmj.332.7552.1259. 
4- Malone ML, Gennis V, Goodwin JS. Characteristics of diabetic ketoacidosis in older versus younger adults. J Am Geriatr Soc. 1992 Nov;40(11):1100-4. doi: 10.1111/j.1532-5415.1992.tb01797.x

5- Umpierrez G, Korytkowski M. Diabetic emergencies - ketoacidosis, hyperglycaemic hyperosmolar state and hypoglycaemia. Nat Rev Endocrinol. 2016 Apr;12(4):222-32. doi: 10.1038/nrendo.2016.15.

6- Hara JS, Rahbar AJ, Jeffres MN, Izuora KE. Impact of a hyperglycemic crises protocol. Endocr Pract. 2013 Nov-Dec;19(6):953-62. doi: 10.4158/EP13077.OR.

7- Kitabchi AE, Umpierrez GE, Miles JM, Fisher JN. Hyperglycemic crises in adult patients with diabetes. Diabetes Care. 2009 Jul;32(7):1335-43. doi: 10.2337/dc09-9032.

8- Laliberte B, Yeung SYA, Gonzales JP. Impact of diabetic ketoacidosis management in the medical intensive care unit after order set implementation. Int J Pharm Pract. 2017 Jun;25(3):238-243. doi: 10.1111/ijpp.12346.

9- Fusco N, Gonzales J, Yeung SY. Evaluation of the treatment of diabetic ketoacidosis in the medical intensive care unit. Am J Health Syst Pharm. 2015 Dec 1;72(23 Suppl 3):S177-82. doi: 10.2146/ sp150028.

10- Singh RK, Perros P, Frier BM. Hospital management of diabetic ketoacidosis: are clinical guidelines implemented effectively? Diabet Med. 1997 Jun;14(6):482-6. doi: 10.1002/(SICI)10969136(199706)14:6<482::AID-DIA371>3.0.CO;2-A.

11- American Diabetes Association. Standards of medical care in diabetes-2015 abridged for primary care providers. Clin Diabetes. 2015 Apr;33(2):97-111. doi: 10.2337/diaclin.33.2.97

12- Devalia B. Adherance to protocol during the acute management of diabetic ketoacidosis: would specialist involvement lead to better outcomes? Int J Clin Pract. 2010 Oct;64(11):1580-1582. doi: 10.1111/j.1742-1241.2010.02348.x.

13- Ullal J, Aloi JA, Reyes-Umpierrez D, Pasquel FJ, McFarland R, Rabinovich M, et al. Comparison of Computer-Guided Versus Standard Insulin Infusion Regimens in Patients With Diabetic Ketoacidosis. J Diabetes Sci Technol. 2018 Jan;12(1):39-46. doi: 10.1177/1932296817750899.

14- Ziegler A, Williams T, Yarid N, Schultz DL, Bundock EA. Fatalities Due to Failure of Continuous Subcutaneous Insulin Infusion Devices: A Report of Six Cases. J Forensic Sci. 2019 Jan;64(1):275-280. doi: 10.1111/1556-4029.13841.

15- Dhatariya KK, Nunney I, Higgins K, Sampson MJ, Iceton G. National survey of the management of Diabetic Ketoacidosis (DKA) in the UK in 2014. Diabet Med. 2016 Feb;33(2):252-60. doi: 10.1111/ dme.12875.

16- Usman A, Makmor Bakry M, Mustafa N, Rehman IU, Bukhsh A, Lee SWH, Khan TM. Correlation of acidosis-adjusted potassium level and cardiovascular outcomes in diabetic ketoacidosis: a systematic review. Diabetes Metab Syndr Obes. 2019 Aug 6;12:1323-1338. doi: 10.2147/DMSO.S208492.

17- Volkova NB, Fletcher CC, Tevendale RW, Munyaradzi SM, Elliot S, Peterson MW. Impact of a Multidisciplinary Approach to Guideline Implementation in Diabetic Ketoacidosis. American Journal of Medical Quality. 2008;23(1):47-55. doi:10.1177/1062860607311015

18- Casuriaga A, Giachetto G, Gutiérrez S, Martínez V, García A, Martínez F, et al. Auditoría de historias clínicas: una herramienta de evaluación de la calidad asistencial. Hospital Pediátrico - Centro Hospitalario Pereira Rossell. Arch Pediatr Urug. 2018; 89(4): 242-250.

19- Uruguay. Ministerio de Salud Pública. Historia Clínica Electrónica Nacional [sede Web]. Montevideo: MSP; 3 de julio de 2019 [actualizada 3 de julio de 2019; acceso 9 de junio de 2020]. Disponible en: https://www.gub.uy/ministerio-salud-publica/tramites-y-servicios/servicios/historia-clinica-electronicanacional

\section{Aporte de cada autor al trabajo}

Santiago Cabral: concepción y diseño del trabajo, análisis e interpretación de datos, análisis estadísticos, redacción del manuscrito.

Noelia Speranza: concepción y diseño del trabajo, análisis e interpretación de datos, redacción y revisión crítica del manuscrito.

Stephanie Viroga: concepción y diseño del trabajo, análisis e interpretación de datos, redacción y revisión crítica del manuscrito.

Gustavo Tamosiunas: concepción y diseño del trabajo, redacción y revisión crítica del trabajo. Ernesto Balverde: concepción y diseño del trabajo, recolección de datos, análisis e interpretación de los datos. 
Soledad Herrera: concepción y diseño del trabajo, recolección de datos, análisis e interpretación de los datos.

Leticia González: concepción y diseño del trabajo, recolección de datos, análisis e interpretación de los datos.

María Eugenia Hernández: concepción y diseño del trabajo, recolección de datos, análisis e interpretación de los datos.

María Joaquina Goldaraz: concepción y diseño del trabajo, recolección de datos, análisis e interpretación de los datos. 